\title{
Untersuchung der Schichtdickenänderung dicker Polymerschichten geeignet für kapazitive Gassensoren
}

\author{
Ulrike Altenberend, Alexandru Oprea, Nicolae Barsan, Udo Weimar
}

Institut für Physikalische Chemie, Universität Tübingen, Auf der Morgenstelle 15, 72076 Tübingen, Deutschland

\section{Zusammenfassung}

Polymerschichten von mehreren $\mu \mathrm{m}$ Schichtdicke, geeignet für kapazitive Gasdetektion, wurden auf reflektierenden Silizium-Substraten hergestellt und untersucht. Der Einfluss der Gassorption auf die Dicke der Polymerschicht, die neben der Änderung der Dielektrizitätskonstante, auch zum kapazitiven sensorischen Effekt beiträgt, wurde mittels einer neuen optischen Methode (optisches Profilometer auf Basis von chromatischer Aberration) festgestellt und quantifiziert. Anhand der erhaltenen Ergebnisse wurde eine FloryHuggins-Sorptionsisotherme bestimmt und der Flory-Huggins-Wechselwirkungsparameter ausgewertet. Die Methode erlaubt den Analytgehalt durch Änderung der Schicktdicke zu bestimmen.

\section{Einleitung}

Verschiedene Polymere wurden im Hinblick auf ihre Eignung als empfindliche Polymerschicht für kapazitiven Gassensoren untersucht [1], [2]. Bei dieser Art von Sensoren tragen zwei Haupteffekte zum kapazitiven Sensorsignal bei: zum einen die Änderung der relativen Dielektrizitätskonstante, bedingt durch Absorption eines Analyten mit Dipolmoment ungleich Null in die Polymerschicht, und zum anderen die Änderung des Volumens (Schwellung) der empfindlichen Polymerschicht im Bereich der KondensatorFeldlinien [3]. Da es sich bei Beiden um Volumeneffekte handelt, sind dickere Schichten von Vorteil. Zur Erhöhung der Selektivität ist es von Interesse die Beiträge dieser Effekte zu bestimmen.

Für diesen Zweck wurde eine neue Methode entwickelt und angewendet, um die Schichtdickenänderung dicker Polymerschichten zu quantifizieren, weil die bekannten optischen Methoden, wie Ellipsometrie oder Optical Waveguide Spectroscopy (OWS), nur für dünne Polymerschichten geeignet sind.

Die Methode benutzt ein optisches Profilometer auf Basis von chromatischer Aberration. Sie erlaubt das berührungslose Scannen einer reflektierenden Oberfläche und damit die direkte Bestimmung der Schichtdickenänderung unter Arbeitsbedingungen (Operando). Bei der Auswahl hat die vorherige Erfahrung mit dem Einsatz der Weisslicht-Interferenz-Spektroskopie geholfen: hierbei wird die Schichtdickenänderung bei dünnen Polymerschichten unabhängig von der Änderung der relativen Dielektrizitätskonstante bei Analytabsorption gemessen, wie von Gauglitz et al. [4] gezeigt wurde.

\section{Experimenteller Aufbau}

Die Probe besteht aus einer empfindlichen Polymerschicht (Polyetherurethan (PEUT), Polyvinylpyrrolidon (PVP) und Polyacrylsäure (PAA)), die über Lösungsmittel-basierte Beschichtungsverfahren wie das DropCoating und Spray-Coating auf ein Silizium-Substrat beschichtet wurde.

Die Messanordnung beinhaltet ein optisches Profilometer (MicroProf ${ }^{\circledR}$ mit optischem Sensor CHR150N), eine für Operando-Messungen geeignete Messkammer und eine Gasmischanlage zur Dosierung der Analyte (siehe Bild 1). 


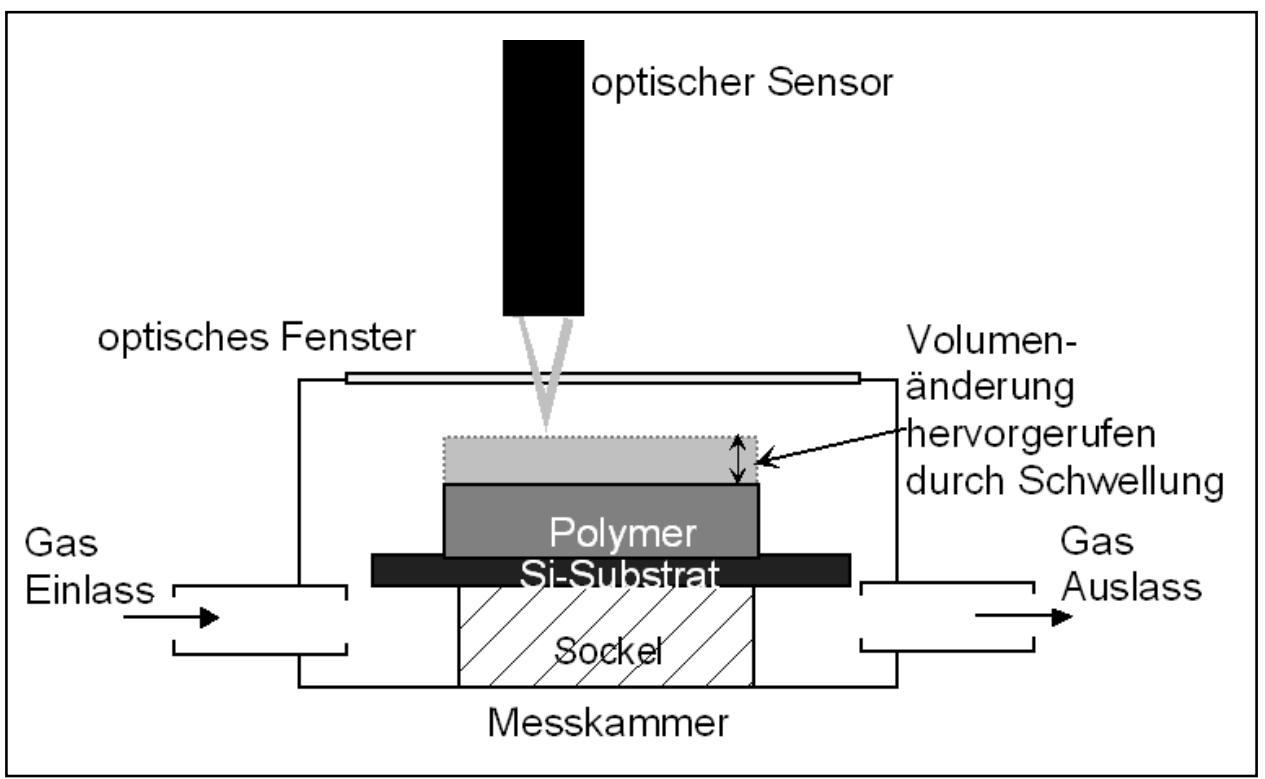

Bild 1 Schematische Darstellung der Messkammer mit Probe und dem optischen Sensor zur Bestimmung der durch Volumenänderung hervorgerufenen Schwellung der Polymerschicht.

Zur Bestimmung der Schichtdicke und der Rauheit wurde die Oberfläche der empfindlichen Polymerschicht unter kontrollierter Atmosphäre (Analyt in synthetischer Luft) mit dem optischen Sensor abgerastert. Die Probe wurde zuerst mit trockener synthetischer Luft gespült und anschließend mit stufenweise zunehmender Konzentration an relativer Feuchtigkeit begast.

Die durch Analytexposition resultierende Schichtdickenänderung $(\not L)$ der empfindlichen Polymerschicht (Differenz der Schichtdicke mit Analyt $\left(L_{c(\text { Analyt })}\right)$ zur Schichtdicke ohne Analyt $\left(L_{0}\right)$ ) wurde bei Raumtemperatur in Abhängigkeit von der gegebenen relativen Feuchtigkeit ausgewertet.

$$
\Delta L=L_{c(\text { Analyt })}-L_{0}
$$

\section{Ergebnisse und Diskussion}

Die Messungen bei den Polymeren PEUT und PVP (siehe Bild 2 und Bild 3) zeigen, dass die Methode geeignet ist, um die Schwellung dicker Polymerschichten im Bereich von wenigen Nanometern reproduzierbar zu bestimmen.

PEUT (siehe Bild 2) zeigt eine mittlere Rauheit der Polymeroberfläche von $21 \mathrm{~nm}$, eine Schichtdicke von $23,5 \mu \mathrm{m}$ und bis zu $80 \%$ relativer Feuchtigkeit eine lineare Zunahme der Schichtdicke. Die absolute Schichtdickenänderung beträgt dabei $250 \mathrm{~nm}$. Man beobachtet eine relative Schichtdickenänderung von einem Prozent bei einer relativen Feuchtigkeit von etwa 80 \%, jedoch ist die Änderung eines entsprechenden kapazitiven Sensorsignals zwischen 5 und $20 \%$. Bezogen auf dieses Beispiel (PEUT), hat der Effekt der Schwellung nur einen sehr geringen Einfluss auf das kapazitive Sensorsignal.

Nach Manoli et al. [5] entspricht die Volumenzunahme, bedingt durch die Absorption von Analyt, dem Volumenanteil ( $\left.\Phi_{1}\right)$ von Analyt in der Polymerschicht.

Der Vergleich der für das Polymer PVP erhaltenen Ergebnisse (siehe Bild 3) mit in der Literatur berichteten Daten zum Wassergehalt in PVP (خ) [6] zeigt, dass die Größe der Schwellung durch die Absorption von Analyt in die Polymermatrix bestimmt wird. Daraus folgt, dass die Untersuchung der empfindlichen Polymerschicht mittels eines optischen Profilometers verwendet werden kann, um den Analytgehalt im Polymer zu bestimmen.

Der Verlauf der relativen Schichtdickenänderung (Schwellung) mit der Konzentrationsänderung folgt im Allgemeinen einer Flory-Huggins-Isotherme, wie auch Biesalski et al. [7] berichtet haben.

$$
\ln \frac{p}{p^{0}}=\ln \Phi_{1}+\left(1+\Phi_{1}\right)+\chi\left(1+\Phi_{1}\right)^{2}
$$




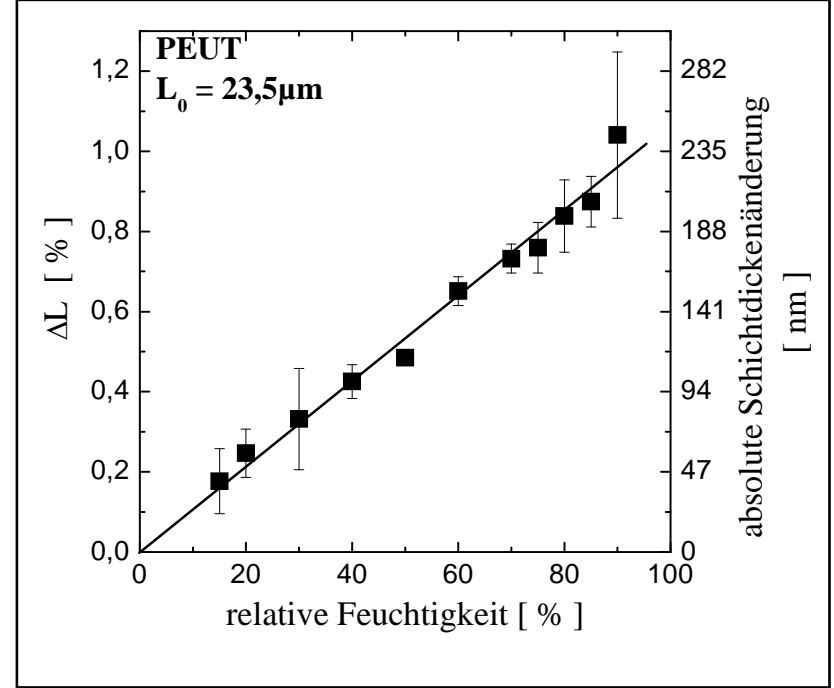

Bild 2 Die relative und absolute Schichtdickenänderung für das Polymer Polyetherurethan (PEUT) in Abhängigkeit von der relativen Feuchtigkeit. Die Schichtdickenänderung $(\Delta L)$ erfolgt linear mit der Konzentration.

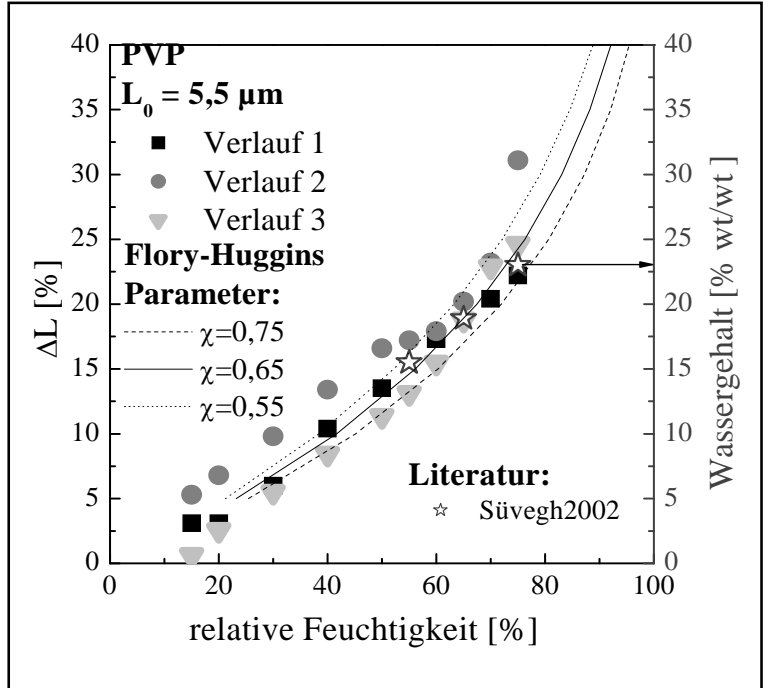

Bild 3 Verlauf der relativen Schichtdickenänderung für das Polymere PVP in Abhängigkeit von relativen Feuchtigkeit. Als Referenz ( $\forall$ ) ist der von Süvegh et al. [6] im Polymer bestimmte Wassergehalt gegeben. Die Linien zeigen die Simulation der Flory-HuggingsSorptionsisotherme.

Dabei entsprechen $\Phi_{1}$ dem Volumenanteil von Analyt in der Polymermatrix, $p^{0}$ dem Sättigungsdampfdruck, $p$ dem gegebenen Dampfdruck und $\chi$ dem Flory-Huggins-Wechselwirkungs-Parameter. Die Simulation der Absorptionsisotherme für das Polymer PVP ergab einen Flory-Huggins-Wechselwirkunsparameter von $\chi=0,65 \pm 0,1$. Die Abweichung zu dem in der Literatur berichteten Wert von $\chi=0,85 \pm 0,1$ [7] kann durch die unterschiedliche Herstellungsweise und daraus resultierend unterschiedliche Dichte der Polymermatrix herrühren.

Im Bereich kleiner Konzentrationen erhält man einen linearen Zusammenhang, wie bei der erwarteten Henry-Sorptionsisotherme anzutreffen wäre.

\section{Fazit}

Die experimentellen Ergebnisse bestätigen die Eignung des optischen Profilometers zur direkten Bestimmung der Schichtdicke und der Schichtdickenänderung dicker Polymerschichten und ermöglichen dadurch ein besseres Verständnis der Gasabsorptionsprozesse.

\section{Literatur}

[1] A. Oprea, J. Courbat, N. Bârsan, D. Briand, N. F. De Rooij, and U. Weimar, "Temperature, humidity and gas sensors integrated on plastic foil for low power applications,” Sensors and Actuators, B: Chemical, vol. 140, pp. 227-232, 2009.

[2] A. Oprea, N. Bârsan, U. Weimar, J. Courbat, D. Briand, and N. F. De Rooij, "Integrated temperature, humidity and gas sensors on flexible substrates for low-power applications," in Proceedings of IEEE Sensors, 2007, pp. 158-161.

[3] A. M. Kummer, A. Hierlemann, and H. Baltes, "Tuning sensitivity and selectivity of complementary metal oxide semiconductor-based capacitive chemical microsensors,” Analytical Chemistry, vol. 76, no. 9, pp. 2470-2477, 2004. 
[4] W. Nahm and G. Gauglitz, "Thin polymer films as sensors for hydrocarbons," GIT Fachzeitschrift für das Laboratorium, vol. 34, pp. 889-893, 1990.

[5] K. Manoli, D. Goustouridis, S. Chatzandroulis, I. Raptis, E. S. Valamontes, and M. Sanopoulou, "Vapor sorption in thin supported polymer films studied by white light interferometry," Polymer, vol. 47, no. 17, pp. 6117-6122, 2006.

[6] K. Süvegh and R. Zelkó, "Physical Aging of Poly(vinylpyrrolidone) under Different Humidity Conditions,” Macromolecules, vol. 35, no. 3, pp. 795-800, 2002.

[7] M. Biesalski and J. Rühe, "Swelling of a Polyelectrolyte Brush in Humid Air,” Langmuir, vol. 16, no. 4, pp. 1943-1950, 2000. 